\title{
THE ELASTIC CONSTANTS OF SPRUCE AS INFLUENCED BY MOISTURE.
}

\author{
BY H. CARRINGTON, B.SC. (VICT.), M.SC. (TFCH.), A.F.R.Ä̈.s.
}

\section{Introduction.}

Previous investigations on the effect of moisture on the elastic constants of spruce have been confined to its effect on Young's modulus in the direction of the grain. The most important work in this connection is probably that of $\mathrm{H}$. $\mathrm{D}$. Tiemann,* who deduced curves for pine spruce and chestnut showing that Young's modulus along the grain decreased as the moisture increased up to the fibre saturation point, and that further addition of moisture had little or no effect on the value of the modulus. Tiemann thus found that the modulus decreased to a minimum at the fibre saturation point and then remained nearly constant and independent of any further addition of moisture. The reason for the independence of the modulus on the moisture beyond the fibre saturation point becomes evident if it is considered that when the fibres are saturated, any further moisture added is retained in the cells and in all probability cannot further affect the stiffness and strength of the wood.

It is proposed in this paper to give the results of a series of experiments made to determine the effect of moisture on the elastic constants of spruce, the constants being determined on the assumption that the spruce has three planes of elastic symmetry. Some consideration will also be given to the relation between shrinkage and moisture and to the effect of continued drying on the shrinkage and moisture and consequent possible damage to the cell walls.

\section{Notation.}

Let the direction of the grain of the tree be denoted by $Z O Z$, the direction perpendicular to this and intersecting the axis of the tree by $X O X$ and the direction normal to $Z O Z$ and $X O X$ by $Y O Y$. Thus, the direction $X O X$ is normal to the annual layers, and the direction $Y O Y$ tangential to them. If, therefore, three mutually perpendicularly lines be considered lying in the above three directions and intersecting at a point $O$ situated some distance from the pith of the tree, the lines are the intersections of the three mutually perpendicular planes of symmetry $O Y Z, O Z X$ and $O X Y$ at the point.

Corresponding with direct strain, in any one of the three principal directions, three elastic constants will be involved. For direct strain in the direction $X O X$ will cause lateral strain in the directions $Y O Y$ and $Z O Z$, and thus involve Young's modulus in the direction $X O X$ and two values of Poisson's ratio corresponding respectively with lateral strain in the directions $Y O Y$ and $Z O Z$. Thus, corresponding with the three principal directions there will be three principal values of Young's modulus and six principal values of Poisson's ratio.

Let $E_{\mathrm{x}}, E_{\mathrm{y}}$ and $E_{\mathrm{z}}$ denote Young's modulus in the directions $X O X, Y O Y$ and ZOZ respectively. Also let $\sigma_{y z}, \sigma_{z y}, \sigma_{z x}, \sigma_{x z}, \sigma_{x y}$ and $\sigma_{y x}$ denote the six values of Poisson's ratio where

$r_{y z}=$ (lateral strain in the direction $\left.Z O Z\right) /($ longitudinal strain in the direction $Y O Y$ ).

Further consideration will show that there are also three principal values of the modulus of rigidity corresponding with shear strain along any of the three pairs of principal planes. Let $M_{\mathrm{yz}}, M_{\mathrm{zx}}$ and $M_{\mathrm{xy}}$ denote these values of the

* U.S. Department of Agriculture, Forest Service Circ. Io8, dated August, I907. 
modulus where $M_{y z}$. . denotes the modulus of rigidity corresponding with shear strain along the two principal planes $O Z X$ and $O X Y$, i.e., along the directions $Y O Y$ and $Z O Z$.

\section{The Test Pieces and Experiments.}

The specimens were prepared from one of four baulks of spruce obtained from the R.A.E. and representative of the good average spruce which was available for acroplane manufacture early in the war. The baulks were well seasoned and free from knots and shakes. Typical microphotographs and an end view of one of the baulks have previously been published.* In these publications the baulks were lettered $\mathrm{A}, \mathrm{B}, \mathrm{C}$ and $\mathrm{D}$ and the experiments described in this paper were performed on baulk $\mathrm{D}$.

The ralues of Young's modulus and Poisson's ratio were deduced from flexure experiments. In the case of the determination of $E_{z}$ and either $\sigma_{z x}$ or $\sigma_{z y}$ the test pieces were about 13 in. long, 1 in. wide and $\frac{1}{4}$ in. thick, and were supported on knife edges I 2 in. apart, the load being applied to two other knife colges 8in. apart and symmetrical with respect to the outer knife edges. When the load was applied the portion of the beam between the inner knife edges was evidently under a constant bending moment and the constants were determined from measurements of the longitudinal and lateral curvatures over about $r$ in. lengths situated at the mid-lengths of the beams. When performing an experiment the bending moment was increased by equal amounts at a uniform rate, care being taken not to overstrain the pieces, and, readings proportional to the curvature taken after every increase by a reflection method. $f$ In this way two straight lines werc constructed, the slopes of which represented the longitudinal and lateral curvatures respectively per unit couple. If the length of the beam was in the direction $\angle O Z$ and the width in the direction $X O X$ the experiment yielded values of $E_{\mathrm{z}}$ and $\sigma_{\mathrm{zx}}$, the latter being given by the ratio of the curvatures. The procedure in the determination of $E_{x}$ and $E_{y}$, and the corresponding values of Poisson's ratio was exactly similar, but the specimens were shorter and their breadths and thicknesses proportionately smaller.

The moduli of rigidity were obtained from torsion experiments on prisms of rectangular cross-section. When a prism with its length in the direction $Z O Z$ and its sides parallel to the two planes of symmetry $O Y Z$ and $O Z X$ is twisted about its longitudinal axis two values of the modulus are involved, i.e., $M_{y z}$ and $M_{z x}$ and by making the ratio of the sides of the cross-section suitable it is possible to calculate one or other of the moduli with considerable accuracy. If the thickness of the piece is in the direction $Y O Y$ then $M_{z x}$ can be obtained with an error of less than 0.1 per cent. provided (breadth/thickness) $\sqrt{ }\left(M_{\mathrm{yz}} / M_{\mathrm{zx}}\right)>3 . \ddagger$ Since the ratio $\sqrt{ }\left(M_{\mathrm{yz}} / M_{\mathrm{zx}}\right)$ is approximately unity the breadths and thicknesses of the pieces with their lengths in the direction $Z O Z$ were made about $1 \frac{1}{4}$ in. and $\frac{1}{6}$ in. respectively.

The values of $M_{x y}$ were determined in a similar manner. The length of the prism was in the direction $X O X$ and the breadth and thickness in the directions $Y O Y$ and $Z O Z$ respectively. In order that the error in the value of $M_{x y}$ should be less than 0.1 per cent. then (breadth/thickness) $\sqrt{ }\left(M_{\mathrm{zx}} / M_{\mathrm{xy}}\right)$ should be $>3$ and since $\sqrt{ }\left(M_{2 x} / M_{x y}\right)$ was equal to about 5 then $M_{x y}$ could have been calculated if the cross-sections had been square. The actual dimensions were about Iin. by $\frac{1}{2}$ in. in the directions $Y O Y$ and $Z O Z$ respectively.

The moduli were deduced from torque twist curves, the twist being measured by the usual reflection method of affixing mirrors to the specimens and using telescopes and scales. More detailed accounts of the methods of determining the

\footnotetext{
* Phil. Mag., June, 1921, and May, 1922.

+ Phil. Mag., Feb., I921.

† Phil. Mag., June, 1921.
} 
constants and the rate of loading the specimens will be found in the Phil. Mag. papers quoted.

\section{Method of Varying the Moisture.}

A curve connecting any particular constant with the moisture could have been obtained either by performing experiments on a number of pieces each with a different humidity or by using one piece and varying the humidity between each experiment. The former method was used by Ticmann, but it was decided to adopt the latter in this research in order to avoid the effect of variability in the eolotropy of different pieces. Seven pieces were accordingly prepared and seven series of experiments performed, one corresponding with each piece. The pieces were allowed to remain in the laboratory for about one month after preparation before the first experiments were performed. Their humidity was then about 12 per cent. After the first experiments had been carried out the pieces were dried in an electrically heated stove at a temperature of from roo to 104 degs. C. for from 24 to 48 hours and tested immediately on leaving the stove, and at intervals until they had again absorbed about 2 per cent. of moisture from the atmosphere of the laboratory. They were then placed over water in a sealed ressel at normal temperature and removed for test at intervals. Immersion in water vapour raised the humidity to between 20 and 25 per cent., and in order to increase it well beyond the fibre saturation point the pieces were placed in water. Previous to testing at humidities beyond the fibre saturation point the pieces were suspended in the sealed vessel over water vapour for some time to retard evaporation and thus avoid internal strains and non-uniformity in the distribution of the moisture.

By the above procedure the moisture was varied from nearly zero to well over roo per cent. and experiments were performed at humidities below the fibre saturation both before and after the pieces had been in water. The percentage moisture was defined in every case as

(weight when tested-weight when dry)/(weight when dry) $\times$ roo.

\section{Results of Experiments.}

The results of the experiments can be seen by a glance at Figs. Nos. 1 to 6 , where each result has been plotted against the corresponding moisture. The points in each case lie approximately on two straight lines which intersect in the region of 30 per cent. of moisture, this being the fibre saturation region of the wood. It will be noted that the constants vary considerably between about $O$ and 30 per cent. of moisture, beyond which they remair nearly constant. All the values of Young's modulus, the modulus of rigidity and $\sigma_{z x}$ decrease from about $o$ to 30 per cent. of moisture whilst $\sigma_{x y}, \sigma_{y x}$ and $\sigma_{z y}$ increase within this region.

The relation between values of $\sigma_{x z}, \sigma_{y z}$ and the percentage moisture are not included. These values are very small, being only about 0.02 , and extra precautions during the experiments would have to be taken. Because of this and since the relations could be deduced from the other results the experiments were not undertaken. The relations can be obtained from the three symmetrical equalities

$$
E_{z} / E_{y}=\sigma_{z y} / \sigma_{y z}, \quad E_{z} / E_{x}=\sigma_{z z} / \sigma_{x z}, \quad E_{x} / E_{y}=\sigma_{x y} / \sigma_{y x} .
$$

These equalities have been shown approximately to hold for spruce containing 12 per cent. of moisture and to be equal respectively to $700 S_{y}, 700 S_{x}$ and $S_{y} / S_{x}^{*}$ where $S_{y}$ and $S_{x}$ denote the shrinkages per unit length of dry timber in the directions $Y O Y$ and $X O X$ respectively, corresponding with 12 per cent. of moisture. In the case of the last of the equalities it will be found from a con- 

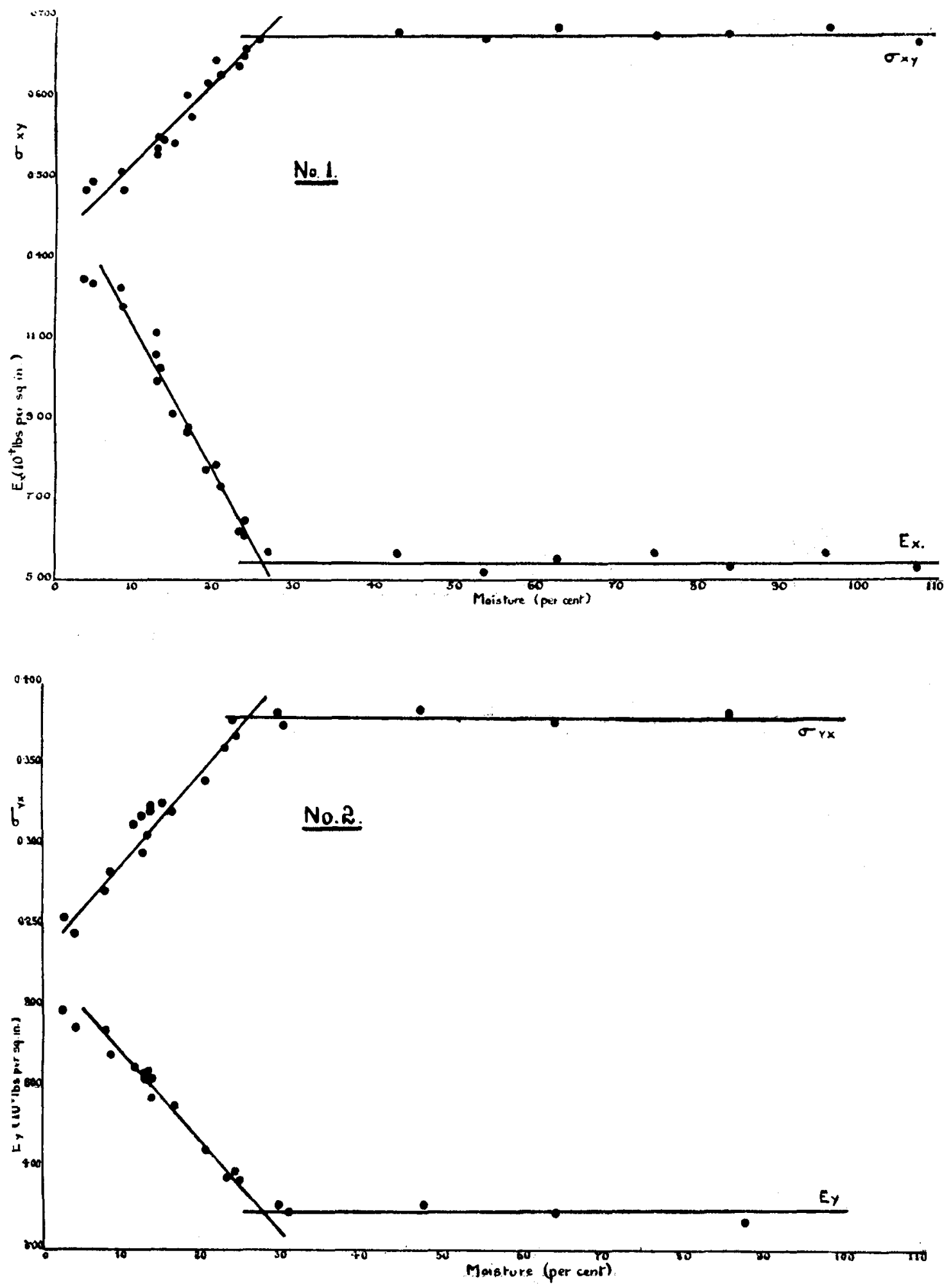
$46(3$

THE HRONHTICAL TOTRNAL

[December, 1922
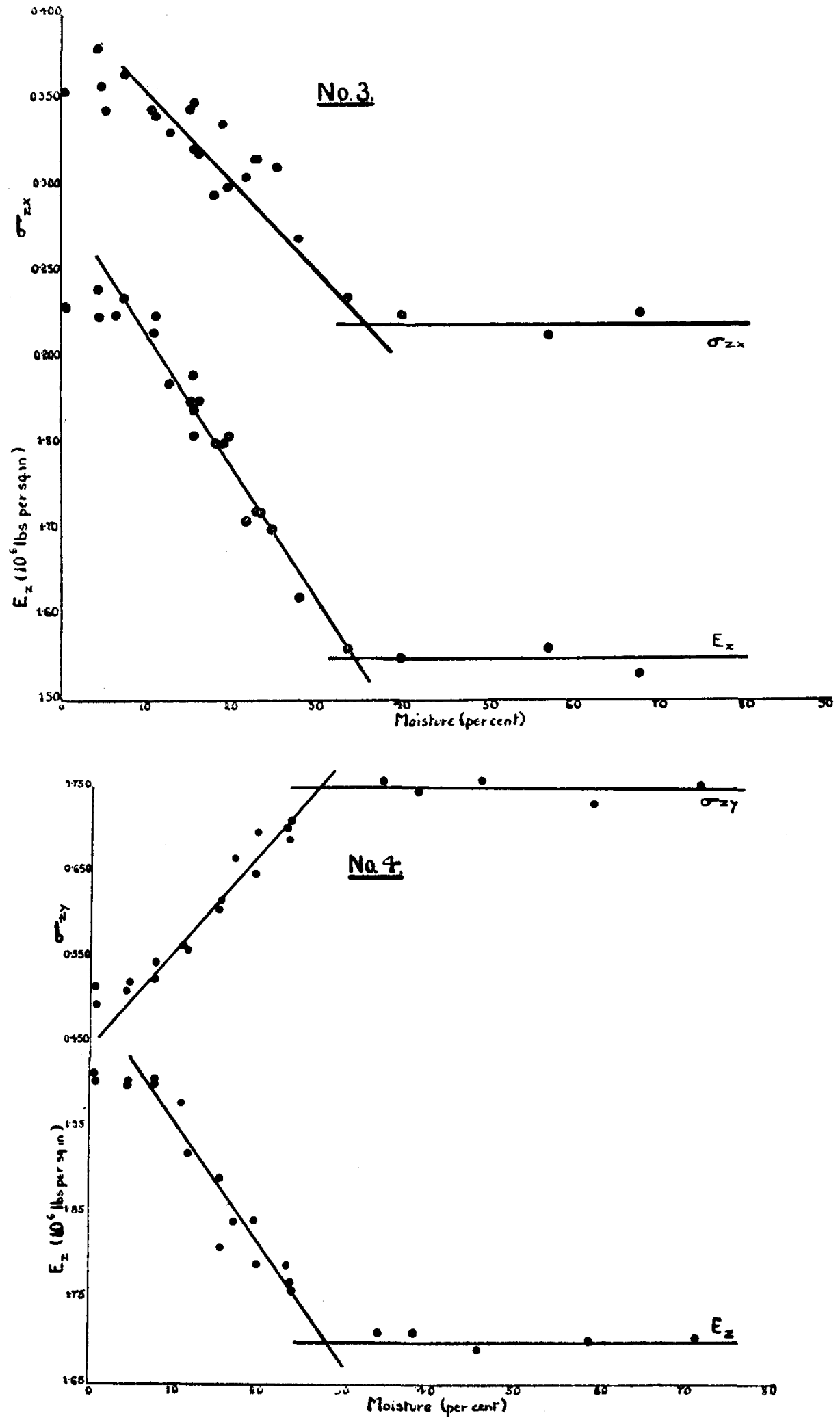

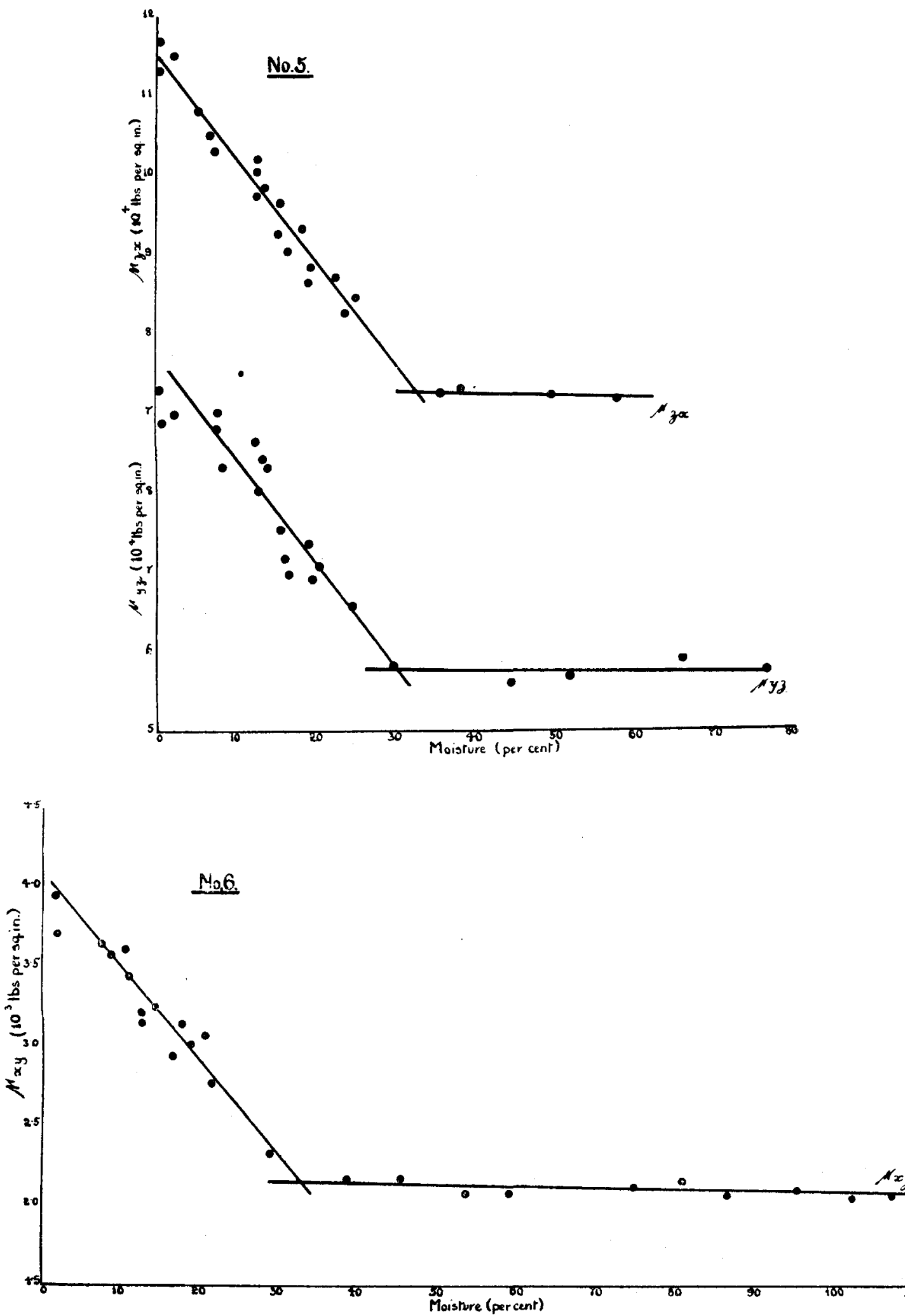
sideration of the curves on Figs. Nos. $\mathrm{I}$ and 2 that previous to the fibre saturation point $E_{\mathrm{x}} / E_{\mathrm{y}}=\mathrm{I} .6_{3}$ and $\sigma_{\mathrm{xy}} / \sigma_{\mathrm{yx}}=\mathrm{I} .73$ so that the ratios are approximately equal. Assuming the other two equalities to hold, the relations between $\sigma_{y z}$ and $\sigma_{x z}$ and the moistures must be similar to those between $\sigma_{z y}$ and $\sigma_{z x}$ shown on Figs. 3 and 4.

The relation between $E_{z}$ and the moisture shown on Figs. 3 and 4 is very similar to that obtained by Tiemann. He, however, finds the relation, between zero moisture and the fibre saturation point, to be best represented by a slightly curved instead of a straight line. This may be due to the fact that in Tiemann's case each plotted value of the modulus corresponding with a particular humidit: was the mean of a number of experiments on different pieces and hence the procedure differed from that adopted in this research.

\section{Relation Between Dimengions (u)xox (2) Yoy and Moisture}

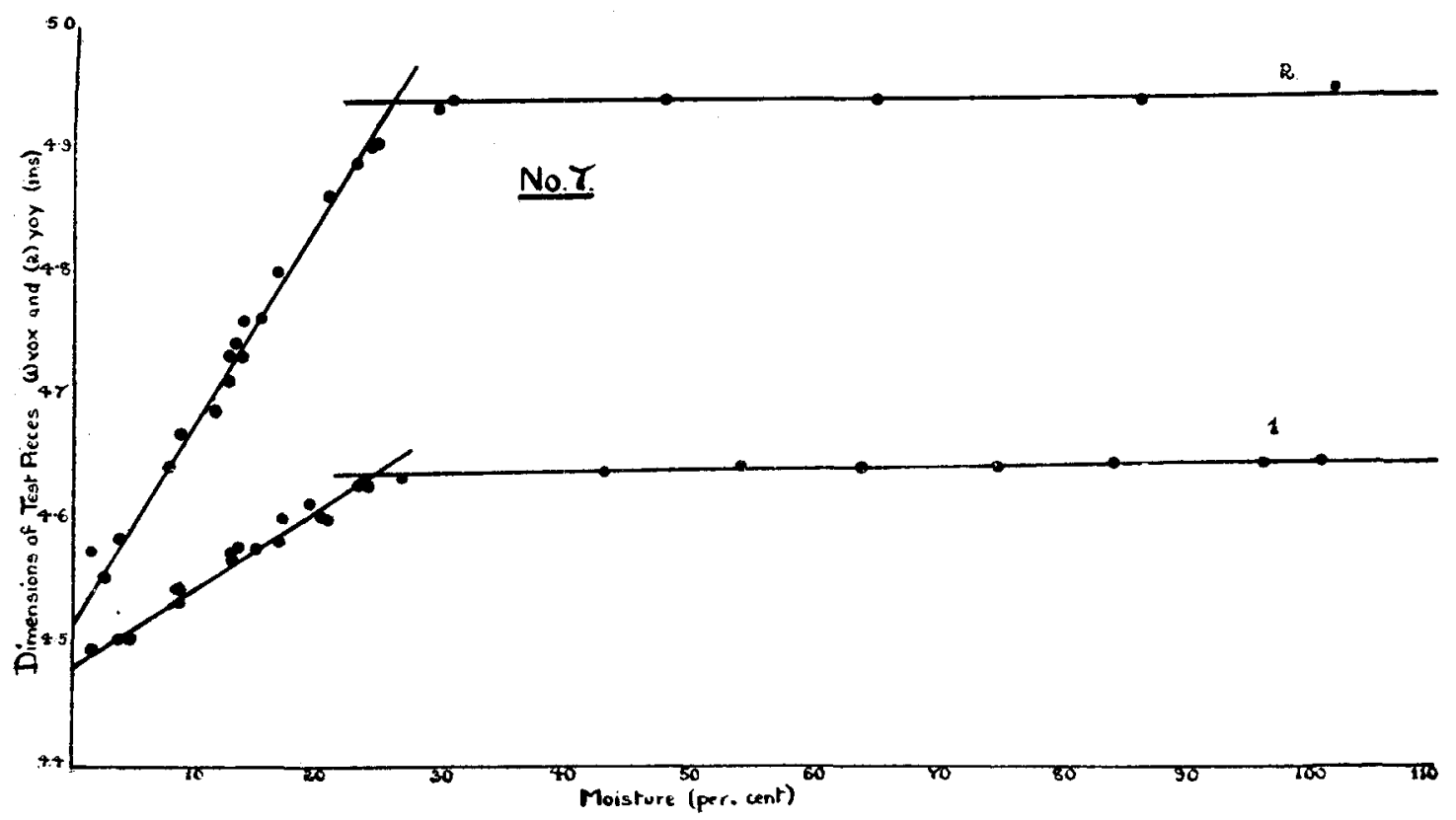

\section{Connection between Shrinkage and Moisture.}

In the case of the test pieces corresponding with Figs. $I$ and 2 the lengths which were in the directions $X O X$ and $Y O Y$ respectively were measured immediately after each weighing. The dimensions were then plotted against the corresponding moistures and resulted in the curves shown in Fig. 7. These are strikingly similar to the curves of Figs. I to 6 and show that the dimensions increase in a linear manner from dryness to the fibre saturation point and then remain constant. It follows that the relations between the shrinkages defined as (dimensions wet-dimensions dry/dimensions dry) and the moisture must be represented in a similar manner. It further follows that the relation between the shrinkage in volume and moisture must also be similar. For let $S_{x}, S_{y}$ and $S_{\mathrm{v}}$ denote the shrinkages in the directions $X O X, Y O Y$ and in the volume respectively per unit lengths and volumes of dry timber then

$$
\left(\mathrm{r}+S_{\mathbf{x}}\right)\left(\mathbf{r}+S_{y}\right)=\mathbf{I}+S_{\mathrm{v}}
$$

therefore $S_{\mathrm{x}}+S_{\mathrm{y}}=S_{\mathrm{v}}$ neglecting the small product $S_{\mathrm{x}} S_{\mathrm{y}}$. 
The shrinkages in the direction $Z O Z$ were very small, being less than o. I per cent. at and beyond the fibre saturation point and were accordingly neglected.

\section{Effect of Continued Drying on Dimensions and Weight.}

The shrinkayes and moisture contents as previously defined are of little value unless the dimensions and weights of the pieces when dry attain to sensibly constant amounts. In order to test this, a number of pieces were prepared and placed in the stove, which was at a temperature of from Ioo to IO4 degs. C., and weighed and measured at intervals. The results in the case of the moisture are shown in Table 1 , and it will be noted that in the case of the large block the moisture increases only slightly. In the case of the small block the increase is sreater and the values are not so consistent. This block was however very small, and owing to convection currents and the rapidity with which it absorbed

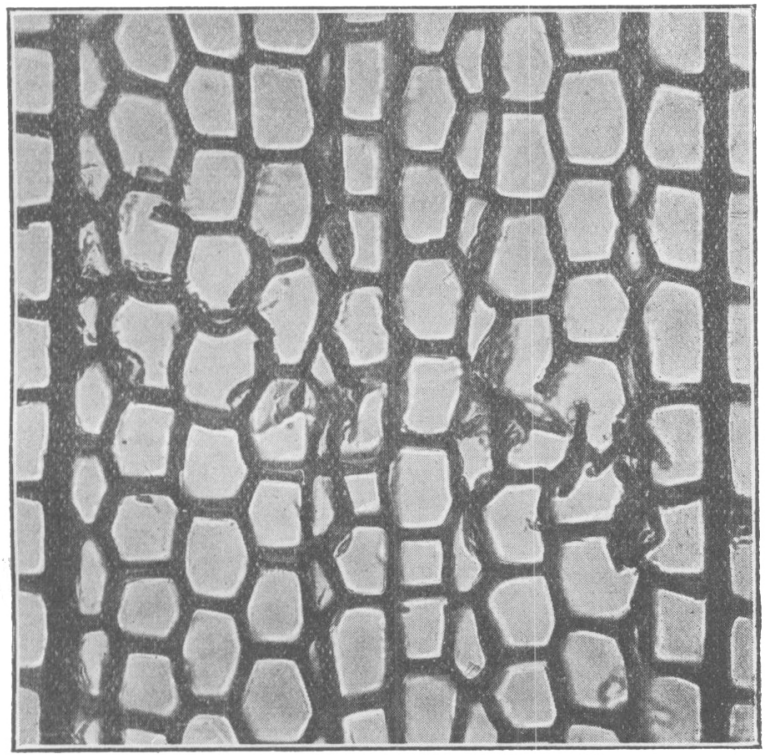

FIG. 8.

moisture from the atmosphere of the laboratory it was difficult to weigh accurately. The increase in the case of the chips and shavings is about the same as for the small block. When considering these increases it is worth noting that cellulose chars slightly at Ioo degs. C., and the increase in the case of the small block, the chips and shavings over that of the large block may be due to the greater surface over volume and the consequent greater loss of weight owing to the charring of the proportionately greater surface.

The shrinkages are shown in Tables 2 and 3 and it will be noted that after a lengthy drying the increases are only slight.

From a consideration of the tables it seems reasonable to assume that after pieces of spruce have been dried at Ioo degs. to Io4 degs. C., for from 24 to 48 hours, they attain to practically constant weights and dimensions so that the shrinkages and moistures are definite properties of the material.

\section{Damage to Cell Walls Due to Drying.}

A consideration of some of the curves in Figs. I to 6 shows that some of the 
points in the region of about o and 5 per cent. of moisture depart from the general linearity of the other points. It may at first appear that this is due to rupture of the cell walls during drying and a consequent weakening of the wood. If this had been so, however, the points would very probably have been-more scattered than is.the case, since each piece was dried at least twice. In order to test the effect of a severe drying on the cell walls a piece was dried at I Io degs. C. for four days and six sections were prepared for examination under the microscope. The cell walls were found to be damagred in the case of one only of the six sections and a photomicrograph of this section magnified about ${ }_{150}$ diameters is shown in Fig. 8. Six sections were also prepared from a piece which had not been dried and no ruptures were evidenced in any one of these sections. A photomicrograph of one of them, also magnified about 150 diameters, is shown in

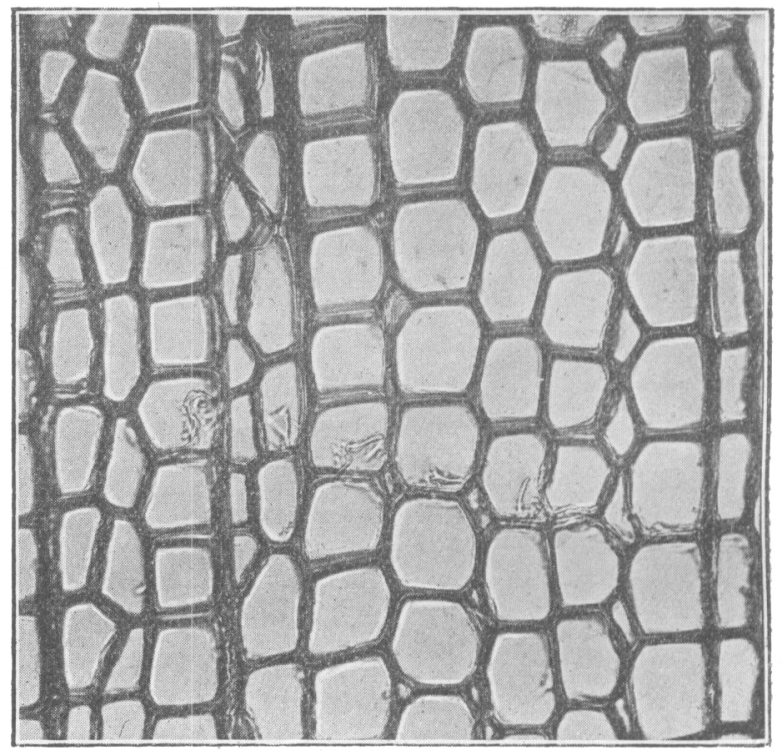

FIG. 9.

Fig. 9. It was therefore concluded that the departure from linearity between o and about 5 per cent. of moisture was not caused by damage to the cell walls due to drying.

Tiemann states that " no matter how dried, wood is found upon re-soaking to be weaker than it was originally." The pieces used by 'Tiemann were, however, generally larger and less homogeneous than those used in this research. If large saturated pieces are dried at Ioo degs. C. or over damage to the cell walls will result and will usually be evidenced by the appearance of cracks. As the dimensions of the pieces are reduced and the drying is conducted more carefully a stage appears to be reached when no damage is evidenced. The pieces used in this research were small and the temperature of the stove was raised gradually over a period of a few hours. Pieces which had been wet were allowed to remain in the laboratory until the moisture fell to about 12 per cent. before they were placed in stove and if subsequent tests had to be performed no piece was allowed to remain in the stove for more than 48 hours at one time.

The writer wishes to thank Mr. H. A. Webb, M.A., for many suggestions and for the loan of Tiemann's paper. 
TABLE 1.

\begin{tabular}{|c|c|c|c|c|}
\hline \multirow{3}{*}{$\begin{array}{l}\text { Time in stove. } \\
\text { hrs. mins. }\end{array}$} & \multicolumn{4}{|c|}{ Initial weights (grams). } \\
\hline & Block. & Block. & Chips. & Shavings. \\
\hline & 84.60 & 2.223 & I 3.292 & I 4.577 \\
\hline 21.20 & $9 \cdot 7$ & $9 \cdot 7$ & 10.2 & 10.6 \\
\hline 46.00 & $9 \cdot 6$ & 9.9 & 10.5 & 10.7 \\
\hline 65.40 & 9.6 & $9 \cdot 7$ & 10.2 & 10.6 \\
\hline $90.5^{\circ}$ & 9.8 & 10.0 & 10.3 & 10.7 \\
\hline 12.55 & 9.8 & IO.I & 10.4 & 10.8 \\
\hline 162.10 & 9.8 & 9.8 & 10.3 & 10.8 \\
\hline 185.20 & 9.8 & $9 \cdot 9$ & 10.4 & 10.7 \\
\hline 257.15 & 9.8 & 10.2 & 10.2 & I I. $x$ \\
\hline 282.00 & 9.8 & I0. 3 & 10.5 & 10.9 \\
\hline $333 \cdot 20$ & 9.8 & IO. I & 10.6 & I I.O \\
\hline $357 \cdot 5^{\circ}$ & $9 \cdot 9$ & 10.0 & 10.7 & I I.I \\
\hline 378.20 & 9.9 & I0. 4 & 10.7 & 11.2 \\
\hline 402.25 & 9.8 & 10.3 & I0.3 & 10.9 \\
\hline $449 \cdot 5^{\circ}$ & $9 \cdot 9$ & 10.6 & 10.7 & I I.2 \\
\hline
\end{tabular}

\section{TABLES 2 and 3.}

Length of piece $7.022^{\prime \prime}$.

Initial Density of piece $26.4 \mathrm{lbs}$./cu. ft.

Dimensions (ins.).

$\begin{array}{cc}X O X . & Y O Y . \\ \text { I.0162 } & 0.283^{2} \\ 0.9965 & 0.2734 \\ 0.9964 & 0.2733 \\ 0.9965 & 0.2732 \\ 0.9963 & 0.2733 \\ 0.9963 & 0.2731\end{array}$

$\begin{array}{cc}S_{x x} \text { 100. } & S_{y x} \text { 100. } \\ 1.98 & - \\ 1.99 & 3.60 \\ 1.98 & 3.65 \\ 2.00 & 3.65 \\ 2.00 & 3.65 \\ & 3.70\end{array}$

Moisture per cent.

Time in stove. hrs. mins.

I I. 9
II.9
I 2.1
I 2.1
I 2.2
0.0 $4^{6.30}$ 7 I.1 5 I 16.25 214.45 $3^{81.25}$

Length of piece $0.3022^{\prime \prime}$.

Initial Density of piece $25.6 \mathrm{lbs}$. $/ \mathrm{cu}$. ft.

Dimensions (ins.).

$$
X O X \text {. }
$$

I. 1945

0.9906

0.9898

0.9882

0.9879

0.9872
YOY.

1.5900

I. 5343

1.5340

I. 5335

r. $533^{\circ}$

I. 5327

$100 S_{x}$
-1.06
2.07
2.09
2.09
2.10

Moisture per cent.

-
I 1.9
I 2.0
I 2.1
I 2.2
I 2.4

Time in stove. hrs. mins.

$$
\begin{array}{r}
0.0 \\
46.35 \\
71.20 \\
116.30 \\
214.35 \\
3^{81.30}
\end{array}
$$

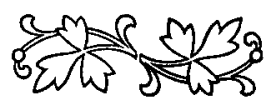

